\title{
A relação entre a não adesão ao tratamento e falsas crenças de pacientes bipolares e seus familiares
}

\section{The relationship between non-adherence to treatment and false beliefs of bipolar patients and their families}

\author{
Karina de Barros Pellegrinelli¹, Mireia C. Roso¹, Ricardo Alberto Moreno² \\ 1 Programa de Transtornos Afetivos (GRUDA), Departamento e Instituto de Psiquiatria do Hospital das Clínicas da Faculdade de Medicina da Universidade de São Paulo (IPq-HCFMUSP). \\ 2 Diretor do GRUDA, Departamento e Instituto de Psiquiatria do HCFMUSP.
}

Recebido: 15/10/2009 - Aceito: 2/12/2009

Pellegrinelli KB, et al. / Rev Psiq Clín. 2010;37(4):183-4

A baixa adesão à medicação entre os pacientes bipolares é conhecida e é um fator importante no insucesso do tratamento de alguns casos. A percepção do paciente a respeito da doença e da importância da medicação influencia na sua adesão' ${ }^{1}$. Sendo assim, quanto mais informados sobre a doença, como ela se manifesta, os tratamentos disponíveis e sua importância para se obter estabilização do quadro clínico, mais aumenta a adesão ao tratamento, levando a melhor desfecho ${ }^{2,3}$.

Fatores psicossociais como personalidade, estilo cognitivo, presença de emoção expressa na família, eventos vitais estressantes e suporte emocional também parecem contribuir em $25 \%$ a $30 \%$ nas alterações no curso da doença, influenciando na recuperação sintomática e na qualidade de vida ${ }^{4,5}$. A baixa adesão ao tratamento medicamentoso ainda é o fator responsável pelo maior número de recaídas. A literatura evidencia que os fatores mais relacionados à baixa recuperação sintomática e funcional são não adesão ${ }^{6}$, presença de sintomas entre episódios ${ }^{7}$, uso de antipsicóticos, baixo funcionamento pré-mórbido, comorbidade com transtorno de personalidade 8 e prejuízo cognitivo relacionado com o transtorno do humor'.

Nos últimos anos, muitos pesquisadores têm se dedicado a avaliar os motivos da baixa adesão ao tratamento, não apenas no que diz respeito ao transtorno bipolar, mas em relação a doenças crônicas em geral. Um estudo feito com pacientes japoneses com doenças crônicas identificou, entre outros tópicos, que a baixa adesão à medicação está associada a crenças pessoais a respeito da doença, não valorização da relação de confiança com o médico, maior valorização dos possíveis efeitos colaterais da medicação e pouca compreensão de sua necessidade ${ }^{10}$.

Bowskill et al. 5 , em 2007, avaliaram 223 pacientes membros do Manic Depression Fellowship (MDF), e aqueles que reportaram baixa adesão à medicação tiveram alta insatisfação com a informação fornecida; já os que reportaram alta adesão tiveram baixa insatisfação com a informação. Isso parece demonstrar a associação entre a informação correta e a boa adesão.

Sendo assim, é importante que a psicoeducação associada ao tratamento do transtorno bipolar (TB) inclua não apenas a informação sobre a doença e seu tratamento, mas leve em consideração as crenças inadequadas dos pacientes que podem atrapalhar na assimilação da informação prestada e consequentemente atrapalhar na adesão ao tratamento e na obtenção de bons resultados. Por isso, o Programa de Transtornos Afetivos do IPq-HCFMUSP realizou um estudo-piloto acerca das principais crenças errôneas dos pacientes e familiares presentes em seus Encontros Psicoeducacionais abertos. Para isso, foi elaborado um questionário que consistia de 32 afirmações (verdadeiras e falsas) a respeito da doença, de seu tratamento, da importância da família e da prevenção de recaídas. Os sujeitos participantes do estudo foram instruídos a atribuir a cada afirmação o critério de verdadeira (V) ou falsa (F). Sessenta e dois sujeitos participaram do estudo. Os resultados demonstraram que $40 \%$ dos sujeitos têm crenças errôneas a respeito da natureza biológica da doença, da importância do apoio da família e dos efeitos da medicação.

As principais crenças errôneas levantadas foram:

1. O TB é um problema psicológico.

2. O tratamento medicamentoso pode comprometer a vida do paciente mais do que melhorá-la (relação risco $\mathrm{x}$ benefício).

3. O TB é emocional, e não biológico.

4. O TB não é um transtorno mental ou uma doença médica.

5. A medicação, além de causar dependência, é prejudicial.

6. A família tem um papel prejudicial no tratamento.

7. A cura é possível.

Essas crenças foram semelhantes às encontradas em outros estudos ${ }^{6,5,1,11}$ com portadores de doenças mentais e crônicas.

Os resultados deste estudo levantam algumas questões importantes a respeito do tipo de informação que precisa ser dada ao paciente e seus familiares a fim de melhorar a adesão ao tratamento:

(a) É importante o esclarecimento a respeito da natureza biológica do TB a fim de separar fatores biológicos dos sociais que podem ser gatilhos para um novo episódio ${ }^{12}$.

(b) Deve ficar clara a ausência de evidência de cura do transtorno, mas a possibilidade de controle dele, como em doenças crônicas em geral. Essa informação pode frustrar pacientes e familiares quando eles percebem que o tratamento é para a vida toda, mas, a partir da aceitação desse fato e havendo uma relação de cumplicidade e confiança entre médico, psicoterapeuta e paciente, o tratamento torna-se mais eficiente.

(c) A família deve ser incluída e orientada desde o início, a fim de que sua participação no tratamento, no controle de recaídas e na melhora da comunicação possa favorecer a manutenção da estabilidade.

(d) Finalmente, é fundamental que se ressalte e esclareça a importância do tratamento farmacológico para a obtenção de bons resultados e os possíveis efeitos colaterais da medicação, bem como as alternativas para amenizá-los.

Esses são pontos cruciais não só para melhorar a compressão da doença e seu tratamento, garantindo maior adesão, mas também para diminuir o estigma e o preconceito que ainda afetam os portadores desse transtorno e seus familiares.

\section{Referências}

1. Hirschfeld RMA, Goodwin FK. Transtornos do humor. In: Talbott J, Hales R, Yudofky S, editors. Tratado de psiquiatria. Porto Alegre: Artes Médicas; 1992, p. 301-28. 
2. Pellegrinelli KB, Roso M, Costa LF, Moreno RA. Impact of psychoeducation in functional and symptomatic recovery in bipolar patients. Bipolar Disord. 2009;11(1):68.

3. Roso MC, Moreno RA, Costa ES. Psychoeducational Intervention on mood disorders: the experience of GRUDA. J Bras Psiquiatr. 2005;27(2):165 [Letter].

4. Bauer MS, Kirk GF, Gavin C, Williford WO. Determinants of functional outcome and healthcare costs in bipolar disorder: a high - intensity follow up study. J Affect Disord. 2001;65:231-41.

5. Bowskill R, Clatworthy J, Parham R, Rank T, Horne R. Patients' perceptions of information received about medication prescribed for bipolar disorder: implications for informed choice. J Affect Disord. 2007;100:253-7.

6. Callahan AM, Bauer MS. Psychosocial interventions for bipolar disorder. Psychiatr Clin North Am. 1999;22(3):675-88.
7. Colom F, Vieta E. A perspective on the use of psychoeducation, cognitivebehavioral therapy and interpersonal therapy for bipolar patients. Bipolar Disord. 2004;6:480-6.

8. Ferrier IN, Stanton BR, Kelly TP, Scott J. Neuropsychological function in euthymic patients with bipolar disorder. Br J Psychiatry. 1999;175:246-51.

9. Gonzáles-Pinto A, Gonzales C, Enjunto S, De Costa BF, Lopez P, Palomo J, et al. Psychoeducation and cognitive-behavioral therapy in bipolar disorder: an update. Acta Psychiatr Scand. 2004;109(2):83-90.

10. Iihara N, Tsukamoto T, Morita S, Miyoshi C, Takabatake K, Kurosaki Y Beliefs of chronically ill Japanese patients that lead to intentional nonadherence to medication. J Clin Pharm Ther. 2004;29(5):417-24.

11. Johnson FR, Ozdemir S, Manjunath R, Hauber AB, Burch SP, Thompson TR. Factors that affect adherence to bipolar disorder treatments: a statedpreference approach. Med Care. 2007;45(6):545-52.

12. Watson S, Young AH. Bipolar disorder: new approaches to psychoterapy. Expert Opin Pharmacother. 2001;2(4):601-12. 